(C) 2017 IEEE. Personal use of this material is permitted. Permission from IEEE must be obtained for all other uses, in any current or future media, including reprinting/republishing this material for advertising or promotional purposes, creating new collective works, for resale or redistribution to servers or lists, or reuse of any copyrighted component of this work in other works. 


\title{
Modelling of Multilayer Biological Medium under Molecular Communication Paradigm
}

\author{
Muneer M. AL-Zubi, Student Member, IEEE, and Ananda Sanagavarapu Mohan, Senior Member, \\ IEEE
}

\begin{abstract}
Molecular communication is an emerging paradigm that enables both the biological and synthetic nanomachines to communicate with each other within an aqueous biological environment such as the communication between living cells. Prediction of the number of drug molecules near a target site, e.g., tumor cells, is very important for determining the required drug dosages to increase positive therapeutic outcomes. In this paper, we derive an analytical expression for the received molecular signal in a multilayer biological environment. We also present development of particle-based simulator and the analytical results for threelayer biological medium compared well with the simulation results. The effect of the diffusion coefficient and the separation distance between the transmitter and the receiver (e.g., targeted cells) are examined.
\end{abstract}

Keywords-drug delivery; diffusion; multilayer; Molecular communication.

\section{INTRODUCTION}

Molecular communication (MC) is a new communication paradigm in which the chemical molecules such as drug molecules diffuse through an aqueous environment inside a human body to perform a specific action at any target site [1]. It is highly appropriate for certain biomedical applications, where the use of electromagnetic (EM) waves is neither possible nor desirable [1]-[2]. In particular, the molecular communication via diffusion (MCvD) is a simple method, which does not require additional infrastructure or external energy. In this, the released molecules by the transmitter diffuse in any arbitrary direction through the biological medium following the Brownian motion to reach a receiver [3]-[4].

In molecular communication by diffusion, the chemical molecules, such as proteins and ions, that contain information are released by a transmitter nano-machine (TN) (e.g., drug delivery device, injection) which then diffuse thorough the biological environment (e.g., blood vessels, tissues) until they reach the target site to be received by a receiving nano-machine ( $\mathrm{RN})$ (e.g., tumor cells etc.,). Hence, the biological medium acts like a communication channel and therefore, modelling of the molecular communication channel between the TN and RN assumes

This work is supported by the UTS FEIT Bluesky Research Grant 2017.

Muneer Al-Zubi and Ananda S. Mohan are with the Centre for Health Technologies, Faculty of Engineering and IT, University of Technology Sydney,NSW, 2007, Australia (e-mail:Muneer.Al-Zubi@student.uts.edu.au, Ananda.Sanagavarapu@uts.edu.au). greater importance in applications such as targeted drug delivery systems (DDS) etc. This helps in the prediction of the received molecular signal (i.e., number of molecules) near the target cells in order to specify the desired amount of drug dosage over time as well as to ensure that the drug concentration does not reach toxic levels within the body [5]-[6]. Molecular communication can also help to improve the efficacy of therapy via targeted drug delivery [3].

There are numerous studies on molecular communication channel in the literature, [7]-[10], but most of them approximate the molecular environment as a single homogeneous region with constant diffusion coefficient. There are some other works [11]-[12] which consider the multilayer molecular medium but only to calculate the average effective diffusion coefficient. Then, the diffusion coefficients of each of the multiple layers are replaced by the average effective diffusion coefficient which then is substituted into the well-known expression for molecular concentration of a single layer medium. However, such average diffusion coefficient is only valid when the diffusion happens along a single axis i.e., one-directional (1-D) diffusion. Also, the averaging model is applicable only under steady state conditions when the time derivative of molecular concentration equals to zero [13]. It cannot accurately model any the actual multilayer biological environment particularly under transient conditions. Inside the human biological environment, many regions with different diffusive properties may exist. Each such region can be considered to be a separate layer with distinct diffusion coefficient and thickness. Some of the examples for such multilayer biological media include transport of drugs across the blood-brain barrier [14] and release of drugs from Drug-Eluting Stent (DES) thorough the layers of coronary artery, i.e., the tunica intima, the tunica media, and the tunica adventitia layers [15].

In this paper, we propose a multilayer channel model for molecular communication via diffusion (MCvD) systems. Our model considers diffusion along any arbitrary direction (3-D) under transient conditions and we derive an analytical expression for the molecular received signal. Furthermore, we also introduce a particle-based simulator for MCvD under multilayer environment for verification with the analytical results. The effect of system parameters, e.g., diffusion coefficients and the separation distance between $\mathrm{TN}$ and RN, on the received signal are examined. To the best of our knowledge, this is the first work in literature that 
consider modelling the transient, MCvD systems with multiple distinct layers.

The rest of this paper is organized as follows. In section II, the system model and the expected received signal, i.e., number of received molecules, for the molecular communication system of multilayer biological environment are presented. The analytical and simulation results for three-layer biological environment and for various values of system parameters are discussed in section III. Finally, the conclusions of our work are highlighted in section IV.

\section{SYSTEM MODEL}

In this work, we consider a point-like transmitting nanomachine $(\mathrm{TN})$ located at any point $\left(\mathrm{x}_{0}, \mathrm{y}_{0}, \mathrm{z}_{0}\right)$ and releases ' $M$ ' chemical drug molecules instantaneously into an isotropic biological environment at time $t=0$. The released molecules diffuse independently in all directions (3-D diffusion) within the biological propagation environment following Brownian motion to reach the receiving nanomachine (RN), which is located at a general targeted position $(x, y, z)$.

The biological environment is assumed to be composed of 'N' layers (regions) each layer with distinct diffusion properties $D_{i}$ and different thickness $L_{i}$. Also, it is assumed that the TN is located in the first layer while the $\mathrm{RN}$ is located in the last layer as shown in Fig. 1. Each layer in the biological environment is assumed to be isotropic, i.e., the diffusion properties to be identical in any direction. We assume that the interfaces between layers are fully permeable where the molecules can move across the interfaces without any physical or chemical reactions i.e., the permeability coefficient has very large value. In addition, we assume that the diffusion is symmetrical (radially) around the $\mathrm{x}$-axis assuming a cylindrical coordinate system.

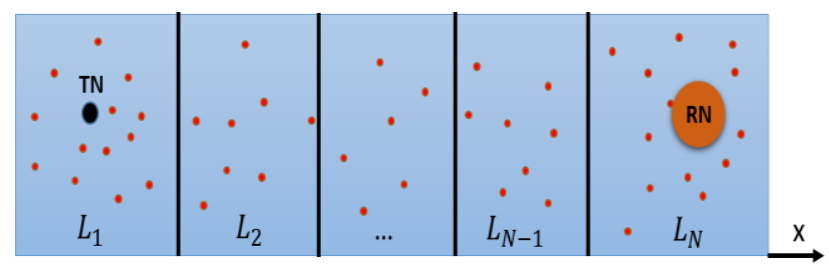

Fig. 1. MCvD model for multilayer biological environment.

Mathematically, the diffusion of molecules that happen independent of each other in a biological medium, is described by Fick's second law of diffusion given by [13]

$$
\frac{\partial C_{i}(x, y, z, t)}{\partial t}=D_{i} \nabla^{2} C_{i}(x, y, z, t)
$$

where, $C_{i}(x, y, z, t)$ is the molecular concentration in $\left(\mathrm{mol} / \mathrm{m}^{3}\right)$ at position $(x, y, z)$ and at time $t$ in $t^{t h}$ layer, and $\nabla^{2}$ is the Laplacian operator.
The parameter $D_{i}$ is the diffusion coefficient of $i^{\text {th }}$ layer in $\left(\mathrm{m}^{2} / \mathrm{s}\right)$ given by Stokes-Einstein equation as

$$
D_{i}=\frac{K_{B} T_{a}}{6 \pi \eta_{i} r_{m}}
$$

where, $K_{B}$ is the Boltzmann constant, $T_{a}$ is the absolute temperature in (Kelvin), $\eta_{i}$ is the dynamic viscosity constant of $i^{t h}$ layer, and $r_{m}$ is the hydraulic radius of the information molecules [10], and $i=1,2, \ldots, \mathrm{N}$.

The TN release " $M$ " molecules instantaneously at a time $t=0$ and at a position $\left(\mathrm{x}_{0}, \mathrm{y}_{0}, \mathrm{z}_{0}\right)$ in layer- 1 represents the initial condition, which is used for solving the diffusion equation (1).

The total solution of the diffusion equation (1) with the boundary conditions at the interfaces can be expressed as a superposition of two functions in Laplace domain [16]:

$$
L\left\{C_{i}(x, y, z, t)\right\}=\delta_{i 1} W(x, y, z, s)+Q_{i}(x, y, z, s)
$$

where, $L\{$.$\} is the Laplace transform operator, W(x, y, z, s)$ is the Laplace transform of $w(x, y, z, t), Q_{i}(x, y, z, s)$ is the Laplace transform of $q_{i}(x, y, z, t)$, and $\delta_{i 1}$ is the Kronecker Delta function given as

$$
\delta_{i 1}= \begin{cases}1, & i=1 \\ 0, & i \neq 1\end{cases}
$$

The function $w(x, y, z, t)$ is the molecular concentration in an isotropic and unbounded medium, i.e., the collisions within the boundaries are elastic. It can be obtained by solving the following diffusion equation, initial condition, and boundary conditions:

$$
\begin{gathered}
\frac{\partial w(x, y, z, t)}{\partial t}=D \nabla^{2} w(x, y, z, t) \\
\lim _{t \rightarrow 0} w(x, y, z, t)=\delta\left(x-x_{0}, y-y_{0}, z-z_{0}\right) \\
\lim _{(x, y, z) \rightarrow \pm \infty} w(x, y, z, t)=0, \quad t \geq 0
\end{gathered}
$$

where, $\delta($.$) is the Dirac delta function.$

The initial condition (6) represents the impulsive emission of the information molecules by $\mathrm{TN}$ at a position $\left(\mathrm{x}_{0}, \mathrm{y}_{0}, \mathrm{z}_{0}\right)$ and at a time $t=0$, and the boundary condition (7) characterizes the boundary condition at the infinity, where the concentration vanishes.

The solution of diffusion equation (5) under the conditions (6)-(7) predict the molecule concentration at location $(x, y, z)$ and at time $t$ due to molecular diffusion of initially emitted molecules at location $\left(x_{0}, y_{0}, z_{0}\right)$ and at time $=0$. This has been widely reported and used in the literature but this is valid only for unbounded medium of a single layer (region) and is given as follows [9], 


$$
w(x, y, z, t)=\frac{1}{(4 \pi D t)^{3 / 2}} e^{-\frac{\left(x-x_{0}\right)^{2}+\left(y-y_{0}\right)^{2}+\left(z-z_{0}\right)^{2}}{4 D t}}
$$

where, $D$ is the diffusion coefficient of a single layer (Layer$1)$.

The molecular concentration $w(x, y, z, t)$ is a spatiotemporal function of both time and distance between transmitter and receiver given by $r^{2}=\left(x-x_{0}\right)^{2}+\left(y-y_{0}\right)^{2}+\left(z-z_{0}\right)^{2}$.

For multilayer medium, the function $q_{i}(x, y, z, t)$ is the solution of the diffusion equation in a cylindrical coordinate system that vanishes at $t=0$, which when it added to (8), satisfies the boundary conditions at the interfaces between layers. The solution requires the inverse Laplace transform of (3) which can be numerically evaluated using Zakian numerical inversion technique [17]. The received signal, which is nothing but the number of received molecules, is equal to the molecular concentration multiplied by both the volume of receiver and number of emitted molecules given by

$N_{\text {mol }}=\frac{2 M V}{t} \sum_{n=1}^{5} \operatorname{Re}\left\{K(n) \int_{0}^{\infty} \beta_{N}(\tau, s) e^{\mu_{N} x} J_{0}(\tau \rho) \tau d \tau\right\}$

where, $\mathrm{V}$ is the volume of the spherical receiver, $J_{0}($.$) is the$ Bessel function of the first kind of order zero, $\beta_{N}(\tau, s)$ is a function that can be obtained by applying the boundary conditions at the interfaces, the radial distance $\rho=$ $\sqrt{\left(y-y_{0}\right)^{2}+\left(z-z_{0}\right)^{2}}, \quad s=a(n) / t$ and the complex constants, $K(n)$ and $a(n)$, are listed in Table I [17]. The expression (9) has been evaluated numerically using MATLAB and accurate numerical results are obtained.

Table I. Complex constants for Zakian method [17].

\begin{tabular}{|c|l|l|}
\hline $\mathrm{n}$ & \multicolumn{1}{|c|}{$a(n)$} & \multicolumn{1}{c|}{$K(n)$} \\
\hline 1 & 12.83767675 & -36902.0821 \\
& $+1.666063445 i$ & $+196990.426 i$ \\
\hline 2 & 12.22613209 & 61277.0252 \\
& $+5.012718792 i$ & $-95408.6255 i$ \\
\hline 3 & 10.9343031 & -28916.5629 \\
& $+8.40967312 i$ & $+18169.1853 i$ \\
\hline 4 & 8.77643472 & 4655.36114 \\
& $+11.9218539 i$ & $-1.90152864 i$ \\
\hline 5 & 5.22545336 & -118.741401 \\
& $+15.7295290 i$ & $-141.303691 i$ \\
\hline
\end{tabular}

\section{NumericAl AND SimUlation Results}

In this section, analytical results, derived in section II, are evaluated numerically and compared with simulation results, obtained via a particle-based simulator for $\mathrm{MCvD}$, which has been implemented in MATLAB. The comparison of results indicate good agreement between simulation and analytical results. For these, we have consider a 3-D diffusion and heterogeneous biological environment with three-layers each layer having different thickness and diffusion properties. For the three layers, the chosen parameters for diffusion coefficients (D) and layer thickness (L) are as follows: $\mathrm{D}_{1}=4000 \mu \mathrm{m}^{2} / \mathrm{s}, \quad \mathrm{D}_{2}=200 \mu \mathrm{m}^{2} / \mathrm{s}, \quad \mathrm{D}_{3}=50 \mu \mathrm{m}^{2} / \mathrm{s}, \quad \mathrm{L}_{1}=5 \mathrm{~mm}$, $\mathrm{L}_{2}=10 \mu \mathrm{m}$, and $\mathrm{L}_{3}=5 \mathrm{~mm}$. The first and third layers have larger thickness (in millimeter range) when compared to the middle layer which allows us to approximate the first and third layers to be unbounded regions when compared with the middle layer. The simulation parameter are chosen as follows (unless stated otherwise): simulation time $\mathrm{T}=4 \mathrm{~s}$, time step $\Delta t=0.1 \mathrm{~ms}$, number of released molecules $\mathrm{M}=10^{5}$, number of iterations 100 , TN located at $\left(x_{0}, y_{0}, z_{0}\right)=(15,5,5) \quad \mu \mathrm{m}, \quad \mathrm{RN}$ located at $(x, y, z)=$ $(-15,1,1) \mu \mathrm{m}$, and the radius of $\mathrm{RN}$ is $2 \mu \mathrm{m}$.

We developed a particle-based simulator and implemented in MATLAB, where the total simulation time $\mathrm{T}$ is divided into many small-time steps $\Delta t$. In this simulation, the positions of information molecules in the environment are tracked at each time step and their positions are updated via random displacements over each spatial axis following the normal distribution $N(0, \sigma)$ with zero-mean and variance $\sigma^{2}=2 D \Delta t$. When the molecules enter a new layer, they will diffuse using the diffusion coefficient of that layer. The receiver is assumed to have the capability of counting number of received molecules at the end of each simulation step and the receiver counter increased by one when each molecule enters the sensing region of the receiver.

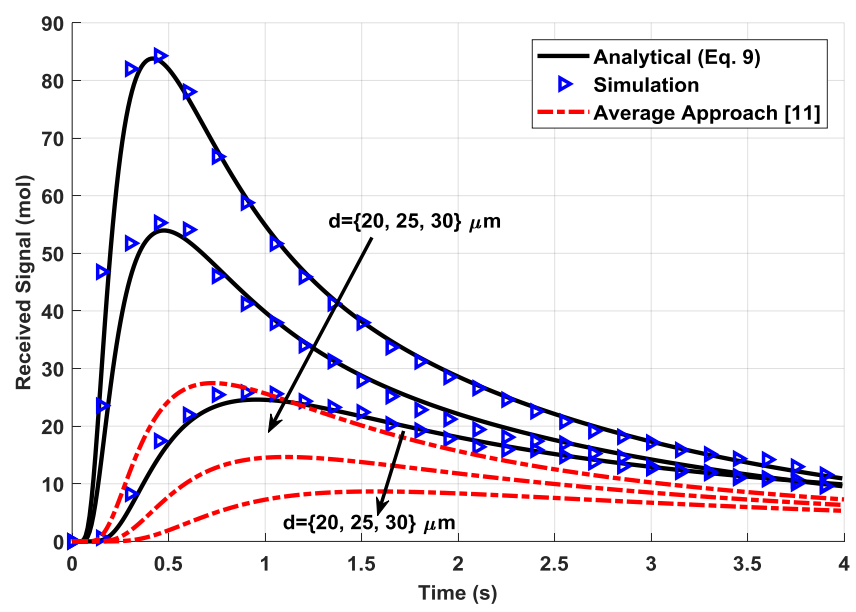

Fig. 2. The received signal as a function of time for different separation distances "d" between TN and RN.

Fig. 2 shows the effect of the separation distance between $\mathrm{TN}$ and $\mathrm{RN}$ on the received molecular signal (i.e., number of received molecules) in three-layer $\mathrm{MCvD}$ environment. As shown in the figure, the received signal amplitude initially shows an increasing trend with time until it reaches the peak value. The time at which peak amplitude occurs is denoted as peak time which is one of the metrics used for characterization. Then, after the peak time, the amplitude starts decreasing. The received signal (including the peak amplitude) decreases with the increasing distance between the TN and RN while the peak time increases. This is due to fewer number of molecules reaching the $\mathrm{RN}$ with the 
increase in the separation distance between TN and RN. The peak time increases because the molecules will take longer time to reach the RN. When average diffusion coefficient for multilayer diffusion is employed [11]-[12], we can observe the larger deviation of the received signal from the actual received signal derived analytically for a three-layer MCvD medium. This shows that the averaging approach can't predict the amplitude and time characteristics of the molecular received signal accurately in a multilayer MCvD medium . However, the analytical results obtained using (9) agree well with the simulation results obtained using our particle-based simulator.

The received molecular signal as a function of time is shown in Fig. 3 as the diffusion coefficient value of layer-2 is varied. The received signal (include the peak amplitude) increases with the corresponding increase in $\mathrm{D}_{2}$ while the peak time decreases. This is due to fact that the molecules entering layer-2 from TN (which is placed in layer-1) will move faster and thus raising their probability of visiting layer-3 (where the target site located) within a shorter time as the diffusion coefficient $\mathrm{D}_{2}$ increases. Thus, potentially larger portion of molecules from layer- 2 will move to layer3 to reach the target site faster when $\mathrm{D}_{2}$ increases. This is the reason why in this case, we obtain larger amplitude and shorter peak time. However, after a specific time as seen in Figs. (2)-(3), the received signals tend to converge to a constant value irrespective of the choice of the simulation parameters. This may be due to the molecules moving far away from the $\mathrm{RN}$ with increasing time and thus only a few number of molecules left to contribute to the received signal. Thus, in this case, the other system parameters have negligible effect on the received signal.

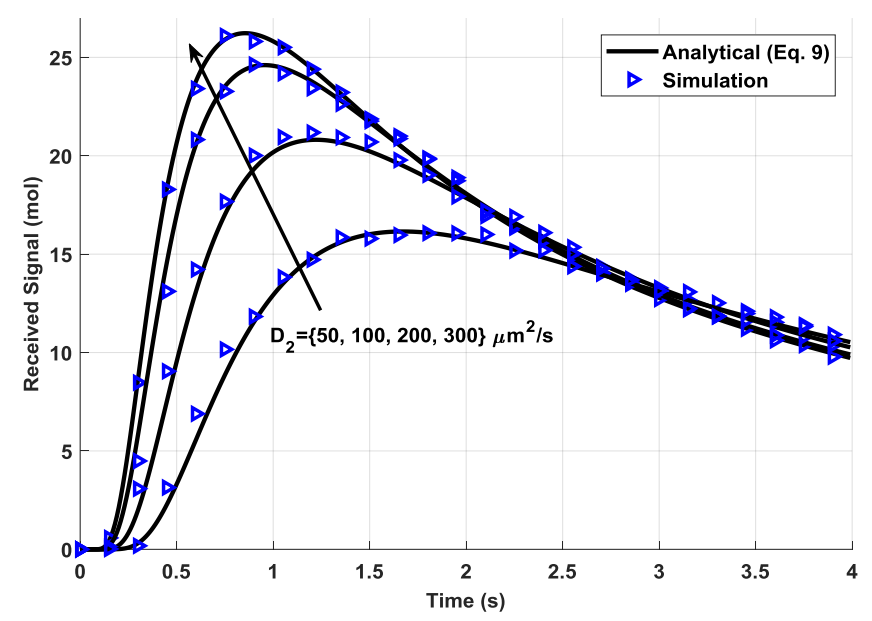

Fig. 3. The received signal as a function of time for various values of the diffusion coefficient $\mathrm{D}_{2}$.

\section{CONCLUSION}

In this work, we have proposed analytical channel model for predicting the molecular concentration in the vicinity of a target site i.e, the receiver, in a multilayered biological environment. Also, we developed a particle-based simulator for molecular communication (MCvD) in a multilayer environment. The simulation results agree closely with the analytical results, for a three-layer environment. We have also examined the effects of some key parameters, viz., the diffusion coefficient and separation distance between transmitter and receiver, on the received signal.

Moreover, it is shown that the effective averaging approach for multilayer medium available in the literature [11]-[12] fails to model the multilayer molecular communication environment accurately. The proposed model has important applications in biomedical engineering, particularly, in the design of future targeted drug delivery system where it can be used for predicting the actual drug concentration around the target site. However, this model can also be used for other applications of molecular diffusion in multilayer medium.

\section{REFERENCES}

[1] I. F. Akyildiz, F. Brunetti, and C. Blázquez, "Nanonetworks: A new communication paradigm," Comput. Netw., vol. 52, no. 12, pp. 2260 2279, Aug. 2008.

[2] N. Farsad, H. B. Yilmaz, A. Eckford, C.-B. Chae, and W. Guo, "A comprehensive survey of recent advancements in molecular communication," IEEE Commun. Surveys Tuts., vol. 18, no. 3, pp. 1887 - 1919, Feb. 2014.

[3] T. Nakano, A. W. Eckford, and T. Haraguchi, Molecular communication, Cambridge, UK: Cambridge Univ. Press, 2013.

[4] P.-C. Yeh, K.-C. Chen, Y.-C. Lee, L.-S. Meng, P.-J. Shih, P.-Y. Ko, W.-A. Lin, and C.-H. Lee, "A new frontier of wireless communication theory: diffusion-based molecular communications," IEEE Wirel. Commun., vol. 19, no. 5, Oct. 2012.

[5] Y. Chahibi, M. Pierobon, S. Song, and I. F. Akyildiz, "A molecular communication system model for particulate drug delivery systems," IEEE Transactions of Biomedical Engineering, vol. 60, 2013.

[6] Y. Chahibi, I. Akyildiz, "Molecular communication noise and capacity analysis for particulate drug delivery systems", Communications IEEE Transactions on, vol. 62, no. 11, pp. 3891-3903, Nov 2014. [7] N.-R.

[7] Kim, A. W. Eckford, and C.-B. Chae, "Symbol interval optimization for molecular communication with drift," IEEE Trans. Nanobiosci., vol. 13, no. 3, pp. 223-229, Sept. 2014.

[8] M. Ş. Kuran, H. B. Yilmaz, T. Tugcu, and B. Özerman, "Energy model for communication via diffusion in nanonetworks," Nano Commun. Net., vol. 1, no. 2, pp. 86-95, Jun. 2010.

[9] D. Kilinc, and O. B. Akan, "Receiver design for molecular communication," IEEE J. Sel. Areas Commun., vol. 31, no. 12, pp. 705-714, Dec. 2013.

[10] M. Pierobon, and I. F. Akyildiz, "A statistical-physical model of interference in diffusion-based molecular nanonetworks," IEEE Trans. Commun., vol. 62, no. 6, pp. 2085-2095, Jun. 2014.

[11] S. M. Mustam, S. Yusof, S. Kamilah, and S. Nejatian, "Multilayer diffusion-based molecular communication," Trans. Emerg. Telecommun. Tech., vol. 28, no. 1, Feb. 2015.

[12] S. M. Mustam, S. K. Syed-Yusof, and S. Zubair, "Capacity and Delay Spread in Multilayer Diffusion-Based Molecular Communication (DBMC) Channel," IEEE Trans. Nanobiosci., vol. 15, no. 7, pp. 599612, Oct. 2016.

[13] J. Crank, The mathematics of diffusion, 2 ed., Oxford, UK: Oxford Univ. Press, 1980

[14] B. K. Lawther, S. Kumar, and H. Krovvidi, "Blood-brain barrier," Cont. Edu. Anaes., Criti. Care \& Pain, vol. 11, no. 4, pp. 128-132, Jun. 2011.

[15] S. McGinty, "A decade of modelling drug release from arterial stents," Math. Biosci., vol. 257, pp. 80-90, Nov. 2014.

[16] N. P. Gaponenko, D. I. Zaks, "The method of images for solving the equations of heat conduction in layered media", Journal of engineering physics, vol.17, no. 3, pp 1162-1166, Sep. 1969.

[17] R. G. Rice and D. D. Do, Applied Mathematics and Modeling for Chemical Engineers, NY: Wiley, pp. 383-385, 1995. 